\title{
The Entrainment of Negation: A Possible Prologue for Interpreting Quantum Mechanics through Light
}

\author{
Timothy Rogers, Timothy.rogers@mail.utoronto.ca \\ Trinity College, revised Oct 12, 2020
}

\begin{abstract}
An exploration of the hypothesis that quantum mechanics is the interpretative framework of relativity theory.
\end{abstract}

Suppose Quantum Mechanics (QM) is a theory of interpretation. Specifically, suppose QM is the interpretative framework for articulating the relational ontology of relativity theory.

Let me clarify. In proposing that QM is a theory of interpretation, I do not simply mean human interpretation. Interpreters can be electrons, or amoebas or scientists. What I do mean is that the implicit conceptual divide in physics between what is present (ontology or "being") and what is represented (epistemology or "meaning") does not fit for QM. And there is a long legacy of research outside the narrow purview of modern physics that suggests this divide does not fit our world either.

In this paper I intend to draw upon this legacy to re-orient questions concerning the foundations of QM. The re-orientation involves relinquishing physical law as a timeless determination of "being" and probing into how it might be that physical laws are instantiated and sustained as laws. That is to ask, what lies beneath or beyond or behind physical laws as their creative origin? Physical laws presuppose objects and their relations. But how does the world come to be parsed into abstracted objects, relations and governing laws in the first place?

The method of the paper is to trace the form of abstraction, the form of bringing into representation. It begins in the intuition or supposition that an identity may obtain between the processes of nature and the processes of human interpretation [Kruse]. The identity is the unknown. There is some sense in which nature and interpretation are the same and there is another sense in which they are different. The sameness must be worked out in relation to the difference as a dialectic. The dialectic holds the two particulars-nature and interpretation-in resonance. Through this resonance, a general form that unites the two particulars might be brought into recognition and abstracted. The presented content thereby becomes synchronously re-presented as harmonic form.

The method of the paper is its meaning.

\section{Harmony}

We begin in relation. We begin by positing an identity between the interior form of interpretationwhat we might call subjectivity-and the exterior form of nature-what we might call objectivity. Let's name this identity "Logic" - from Logos - where the term Logic refers to an abstract indefinite form that 
we intend to bring into determination. Indeed, the process of bringing into determination is what we mean by Logic.

First let me say what we are not going to do. We are not going to bring the term Logic into a pre-existing system of determination, at least not wholly or totally. We are not going to begin with definitions or axioms. In fact, we are going to begin by defining Logic through negation. Namely, Logic is not Rational Understanding.

Drawing from Kant and Hegel [Kerruish and Petersen], by the term "Rational Understanding" $\left(\mathrm{RU}^{1}\right)$ I mean the form that thinking assumes when it operates within a relational system of meaning formation that is rule bound. For example, the form thinking takes in deductive reasoning within Number theory. Within such a system, thinking takes the form of valid reasoning that is constrained by the rules or "laws" and classical or binary logic plays a truth preserving role. "This role is premised on truth definiteness, that is the validity of either-or reasoning as applied to the truth values true and false" [Kerruish and Petersen]. Wittgenstein's Tractatus Logico-Philosophicus, Whitehead and Russel's Principia Mathematica, and Habermas' Theory of Communicative Action are all primarily concerned with $\mathrm{RU}$, for example.

Because RU is an "essential moment of thought" [Kerruish], it is part of Logic. However, Logic (in the sense intended in this paper ${ }^{2}$ ) is more expansive than RU because it also considers the systems and their relations within which RU operates. At the same time as it moves within systems of RU (such as valid modes of deductive reasoning), Logic also moves beneath or beyond or between those systems. In this way of thinking, Logic is more than truth-preserving, it is also generative in truth. Generation or creation happens, for example, when a new concept is first recognized as something that has the possibility of being understood. The process whereby a new concept is recognized and brought into determination as something understood is called abduction or abstraction. With $\mathrm{RU}$, the concepts have already been abstracted by the relational system within which $\mathrm{RU}$ is defined or determined.

Hegel and Kant use the term Reason to implicate this broader notion of thinking unconditioned by systems of understanding. We are not going to do this because it may inadvertently condition our perspective of the beyond of RU to be a human process. Instead, the term Logic seems apt for our purposes. In part, it is apt because it can move between the subjective and the objective. We can think of Logic as a human process of thinking. We can think of Logic as an abstract form of determining that is

\footnotetext{
${ }^{1}$ I am introducing this term and then using an acronym as a matter of method. The term "understanding", as used in common language, is both vague and multi-dimensional in meaning. I want to focus on a more specific aspect of "understanding" that has do with classical (binary) logic and rule-bound reasoning and which I am calling "Rational Understanding" and labelling RU. The term "rational" is introduced by juxtaposition as a pointer to an aspect of understanding. As I focus on this aspect, the concept begins to take on an external, determinate form, but at the same time it loses the inner sense of what is usually meant by "understanding". In other words I am trying to pull out from the experiential notion of "understanding", a concept-image as an external form. The label RU helps to purify reference throughout this process.

${ }^{2}$ More on method. The notion "understanding" is the subject of the current discussion. But as a subject, this notion is "experienced from within" as it were, and is vague and multivalent. The term Logic refers to an external form, but one that I am trying to interrogate and expand. In classical logic, the term Logic is well defined and the interior process is determined by the mechanistic underpinnings of valid reasoning. If that was all that I meant by Logic, then Logic and RU would be the same concept. Instead, however, I have introduced the concept-image RU as a mediator that relates the interior experience of "understanding", namely thinking, with the exterior form of classical logic. This wedge term is intended to open up a gap between inner and outer.
} 
not bound to human thought processes, such as computer logic or the mechanistic manipulation of symbols. We can also think of Logic as the principle behind natural law, such as the law governing the behavior of a system of electrons.

Therefore, let Logic be the term we use to mediate the disjuncture between interior, subjective processes of interpretation and the exterior, objective processes of nature ${ }^{3}$.

Given that we have introduced the term Logic by defining it negatively as Other than or Beyond RU, we also need to be conscious of employing a form of thinking about Logic that is not constrained by the formal systemic structures that characterize RU, such as rule-bound valid reasoning. Logic (in the sense intended in this paper) is more than deductive reasoning and it is also more than classical or binary logic.

Therefore, in a contrapuntal movement, we will also consciously engage in a form of thinking that opposes classical logic, namely harmonic thinking ${ }^{4}$. This form of thinking involves bringing two particulars (in our case particular systems) into relation through an abstract indefinite locus. The relation between the two particulars becomes the Deep Metaphor. Like any metaphor, the two particulars are said to be the same although they are manifestly different. The locus acts a pivot in the movement from one particular to the other and back again in search of a more general abstract form that unites the two particulars. The two particulars that form the Deep Metaphor of this paper will be the systems of Nature and Thought. The locus will be that to which the word Logic points and which is to be brought into determination. Reasoning will move through a series of harmonic progressions in which particular aspects of one system are carried over to the other system as relational movements and then the relational movement is given general form by returning back to the original system at a higher level of abstraction.

Through these harmonic progressions we hope to arrive at a new and more general understanding of both logic and determination.

Further reading: Light Signifying Form: Peirce on creativity, responsiveness and emergence in quantum, biological and linguistic systems.

\footnotetext{
${ }^{3}$ Further note on method. The goal is to use the interior processes of thinking to expand the external, formal aspects of Logic and then apply this expanded exterior form back upon the question of the interpretation of quantum mechanics. There is a reversal involved, such that when we apply the exterior form back onto physical systems we are no longer speaking about human thinking. Thus, in the movement of this discussion, exteriority is governed by form and interiority is governed by metaphor.

${ }^{4}$ Harmonic thinking is the logical complement of deductive reasoning. Whereas deductive reasoning is informed by differentiation through the binary division of the excluded middle (either/or), harmonic thinking is informed by integration in which different particulars are harmonized by the identification of a generalized abstract form that is the same for each. Mathematical proofs involve deductive reasoning; metaphors involve harmonic thinking. These two logical complements together constitute the Logic of Two. (Note that what many logicians call "binary logic" is only the determined half of the complement and leaves out of consideration the Other to the same which is harmonic thinking.) The Logic of Two involves complementarity and requires two disjoint "voices" in open relation. The standard "Copenhagen interpretation" of QM uses the Logic of Two. This paper, however, does not use the Logic of Two. Instead, it uses the Logic of Three whereby the disjointed relation of complementarity is mediated by a Third as creative origin or source, namely Light. The inference is that there is a Logic corresponding to each Natural Number that characterizes its essential form. For more on the Logic of Two, see Gallagher. For more on the Logic of Three, see Marion. For more on the relation between the Logic of Two and the Logic of Three, see Levinas.
} 


\section{First Harmonic Progression: Emergence}

If Logic be not RU, then also let Logic be not Law. For if Logic is Law, then Logic is RU.

The negation carries through both aspects of our Deep Metaphor. Just as RU is not the highest category of interiority or subjectivity [Levinas], so Law is not the highest category of exteriority or objectivity [Unger and Smolin].

But if we begin in this radical negation, don't we lose our grounding? Doesn't Logic become an empty sign that might mean anything we choose? Aren't we entering into meaninglessness as the so-called logical positivists have decried?

Maybe. There is a risk. It all depends on how we take this negation or limit. The term Logic has been loosened from its determinate meaning as classical binary logic and is free in the way that a symbol abstracted from a lawful system like Number theory has a free or indeterminate reference in itself. However, the freeing up of the notion of Logic is not being undertaken for itself. The term Logic is first being taken as a zero point or "non-lieu" through which we can turn back upon ourselves to see the Deep Metaphor-Thought : Nature-in a new light. Rather than turning towards meaninglessness, this will hopefully prove to be a turning away from meaninglessness.

With this intention, let us look back upon the Deep Metaphor.

From the vantage of Thought, the intrigue of Number theory has long presented itself as a truth preserving formal system. During the last century, this system was taken up by Russell and others as archetypal for all truth preserving formal systems. The laws governing the system were re-cast as logical rules. The formal basis of these truth-preserving rules was the law of the excluded middle, whereby any validly formed statement about Numbers must be either True or False. Number theory became a mechanistic process of deducing True theorems by following the rules. At the same time, Numbers as numbers, were emptied of any interior meaning. The rules could and were externalized and reproduced in mechanistic systems, such as computers. This program, however, was stopped short by three discoveries regarding its limitations. First, Russell's paradox brought into question the whole foundation of the program in its intention to create a closed system of "truth" - that is to say, the intention to limit, define or contain Truth. As a result, the program's intention became meaningless, as Fregel recognized. Second, Turing and others showed that there are incomputable numbers. The embodiment of the system as real spatio-temporal processes demonstrated the incompleteness of the embodied system. Third, Godel showed that as the signs of Numbers were emptied of their interior meanings, they could be made to point back to the formal system in order to transcend the system. Numbers were revealed to refer to more than the finite understandings that came from the logical rules or laws of the formal system.

So, when laws of thought were taken as the highest category for understanding Numbers, the intention became meaningless, the embodiment was incomplete, and the formal system was transcended.

From the vantage of Nature, the reduction of Newtonian mechanics has ruled the land for generations. Physical systems are taken to be constructed from elemental parts or states. The parts or states exist eternally in themselves. Their relations are governed by deterministic Laws. Systems are nothing more than assemblages of parts and, as systems, they lack wholistic identity. All is laid bare to the human mind. Nature is merely a mechanistic unfolding of a pre-determined process. However, during the last 
century Nature has revealed herself to be quite Otherwise. At the core of the determinate processes in Nature there is a fundamental indeterminism, as manifested, for example, in the Heisenberg Principle of QM. Nature always eludes reduction to finite, discrete elements. Furthermore, relativity theory articulated that assembled parts, as spatial structures, are never synchronized in themselves because all relations are mediated by light. The whole and its constituent parts are categorically different. Finally, the stars above revealed that our world and all it contains was created from a mysterious Beginning. Nature is creative.

So when Nature was assumed to be determined, her body vanished into nothingness, her rules became contingent possibilities, and the great Beginning revealed itself through the mystery of birth.

As we look back in this way on the Deep Metaphor, we can see a parallel process whereby first Law is taken as the highest category, then finite individuals are isolated from relation and emptied of meaning, and finally the whole program folds back on itself in a transcending movement.

This reflective process is what we will mean in this paper by Logic.

Having completed the first harmonic progression, we can now trace a coarse outline of our first general form for that to which the term Logic refers. Namely, the form of emergence. Emergence is a process that begins within a rule-bound system. The process involves kenosis whereby one or more elements of the system are emptied of their significance for the system. Kenosis is followed by return which is a process of fulfillment. The emptied elements become the fulfillment of the meaning or significance of the system as a whole. Fulfillment reveals a new dimension or depth as a gate or door to a realm that transcends the whole system. As a turning point, the gate or door is the mysterion which is the source and fulfilment of the unfolding of depth. Emergence is a lifting up out of the embedded system or body and into the light. Emergence is birth, the mysterious feminine element that appears to have eluded former men of science.

Further reading: On the Embodiment of Space and Time: Triadic logic, quantum indeterminacy and the metaphysics of relativity.

\section{Second Harmonic Progression: Paradox}

Through the first harmonic progression, we touched upon the limits of Rational Understanding by bringing the underlying principle of RU-Law-into relation with its other-Metaphor. Not only did this happen within the discourse, it was also the underlying theme of the first harmonic progression. For it was my intention to introduce a metaphorical relation between the rational understanding of natural numbers that reigned in the time of Pythagoras and the rational understanding of the world that dominates modern physics. And just as the Pythagoreans discovered that there was a mysterious interior limit to rational numbers, and that beyond this limit were irrational numbers such as pi. So too, might we not venture that there is a mysterious interior limit to the "rational" objects of classical physics and that beyond this limit are "irrational" objects. In this venture, "irrational" is to be reclaimed as a

\footnotetext{
${ }^{5}$ Another note on method. In the current context, the term "irrational" begins as pure negation. Irrational is that which is not rational. If the assumption is that only the rational is real, then the term "irrational" in itself is meaningless. It only has meaning in relation to "rational" as some kind of nothingness that exceeds the rational. But to point beyond the rational is to identify something, namely a limit. A limit is not nothing. If the rational is
} 
positive term denoting something that is real but not containable within the formal logic of RU. Irrational does not mean unreasonable, for example. And my offer is that we might move from the rational to the real by expanding our understanding of the form of logic.

$\mathrm{RU}$ is based on the law of the excluded middle, which we shall take to be the principle of law itself. A either is or is not. And what is $A$ if it is? $A$ is $A$. And if $A$ is not, then not-A. The primary relation is identity: $A$ is $A$. Difference is negation: not- $A$. And the negation of negation is a return to identity. $A$ is not not-A. The underlying movement which sustains the being of $A$ actually involves a moment of return: not-not-A is $A$. The death of death is Life.

While RU begins in the Self-identity of the thing called " $A$ ", metaphor begins in the Otherness of relationality. When we say through metaphor that $A$ is $B$ we are saying that $A$ both is and is not $B$. Metaphor is the excluded initiative of the binary logic of RU. It is excluded because, as a proposition, it is a contradiction. Metaphor sublates the Self-identical form-A is A-that grounds binary logic. Metaphor sublates this Self-identical form by calling into question the finitude of $A$ as the ground of itself. Through the movement of metaphor, A relates to something other than itself and so can enter into the plurality of relations that provide the formal basis for its own Selfhood.

RU takes its elements to be given moments of timeless stasis. Each stasis is a particular locus for meaning formation. The identity of stasis is given. Metaphor is the Self-emptying of stasis in order to point to or signify something Other. Otherness manifests through likeness or quality or essence that brings two particulars into unity. Metaphorical identity is relational and draws particulars into common or shared form.

Traditional binary logic works within formal systems that are themselves given. The givenness of the each elemental stasis as a whole within the system is reflected in the givenness of the formal system as a whole. The discrete iterability of a foundational stasis as Self-identical form grounds the system. For example, in the system of Natural numbers the foundational stasis is 1 . Because 1 can be repeated, the particular instances of momentary stasis can be related to a common form, namely, the form of the finite unit. This foundational form is the creative source for other stases as forms, such as the numbers 2, 3 and so on. The foundational stasis, the other stases and the system all share the common quality of being "whole". The movement of binary logic is the working out of the given particular forms while remaining contained within the formal system. Within binary logic the sublated relationality of metaphor might be said to assume the role of effective cause or implication. Whereas stases and systems are spatial, the sublated relationality of metaphor becomes temporal. It manifests the Otherness to spatiality which remains Other and refuses to be subsumed into spatiality. For example, in the system of Natural numbers we might say: 1 plus 1 causes 2 . This causal movement is the temporal form of equality, where equality relates particular stases by reference to common form. Equality is grounded in the Self-identity of the foundational stasis: 1 is 1.

Equality ${ }^{6}$ is the relation of two as stasis through balance or mutual symmetry. Yet it can also contain the movement of metaphor through a process of expansion or growth (in our case the expansive movement

limited, can it still be claimed as the full measure of the real? Perhaps we can postulate a reality beyond rationality by negating the negation. This is the method of the Logic of Three. There is the rational, the irrational which is the Other to the rational, and the limit or boundary between the two which opens to the world.

${ }^{6}$ Equality comes about from the sublation of the Otherness of relationality. As process, this sublation - a coming into equality-could also be called Justice [Levinas]. 
is addition). So we might say that, in traditional binary logic, metaphor empties itself of its pure dynamism in order to create stasis which then allows the formation of a static system or structure of meaning formation, that is to say it creates a formal logical system. The dynamism is given over to the interpreter as the subject who enters into the expansive system and explores its meanings. It is in this sense that one might speak of systemic binary logic as "spatial" and logical reasoning as "temporal".

Is it possible to bring the dynamism of metaphor back into the explicated formalism of logic? At first blush, the answer appears to be no, because while we have been able to translate the "is" of metaphor into equality within formal logic, the "is not" of metaphor leads us into contradiction. Yet suppose we soften the tenor of the relationship. When we say that $A$ is a metaphor for $B$ we don't mean they are identical in every respect, rather we mean that there is something about $A$ which is the same as $B$. A implies $\mathrm{B}$. In doing this, A gives up, so to speak, its totalizing formal identity so that something can be shared with $B$. Let's represent this symbolically as $A \rightarrow B$. If $A$ is true, then $B$ is true. However, the relationship is asymmetrical in the sense that $B$ does not necessarily imply $A$ as would be the case for equality. B is "greater" than A, so to speak. In a movement away from equality, A is diminished as object or image so that $B$ might be expanded as subject or essence.

Now, within a system of binary logic, $A \rightarrow B$ and $A \rightarrow$ not-B cannot both be true because then, by virtue of the identity of $A$ with itself we would have the contradiction that $B$ and not- $B$ were both true. Consider a system where it is true that $A \rightarrow B$. Suppose someone comes along and claims $C \rightarrow A \rightarrow$ not$B$. Would we not have to say that $C$ includes the impossible because if $A \rightarrow B$, then it is impossible that $A$ $\rightarrow$ not-B? Would we not have to say that the claim is meaningless or absurd? This is the "Lewis Carroll problem" analyzed by Florensky in The Pillar and the Ground of Truth.

However, Florensky argues for a different conclusion. To demonstrate his argument, let's take the example of $A=$ rain and $B=$ wet. The starting truth claim is: rain $\rightarrow$ wet. While the second truth claim is that $\mathrm{C} \rightarrow$ rain $\rightarrow$ not-wet. What happens if we take both claims to be true?

$$
\operatorname{rain}[\mathrm{A}] \rightarrow \text { wet }[\mathrm{B}] \quad \text { AND } \quad \mathrm{C} \rightarrow \operatorname{rain}[\mathrm{A}] \rightarrow \text { not-wet }[\text { not- } \mathrm{B}]
$$

The first claim can be inverted by reversing signs: not-wet $\rightarrow$ not-rain. In the second claim, we can replace the implication, rain $\rightarrow$ not-wet, with the disjunction ${ }^{7}$ : not-rain or not-wet.

$$
\text { not-wet [not B] } \rightarrow \text { not-rain [not-A] AND } \quad \mathrm{C} \rightarrow \text { not-rain [not-A] OR not-wet [not-B] }
$$

Inverting the arguments gives:

$$
\mathrm{C} \rightarrow \text { not-rain [not-A] OR not-wet [not-B] AND not-wet [not-B] } \rightarrow \text { not-rain [not-B] }
$$

This can be reduced as follows:

$$
\begin{aligned}
& C \rightarrow \text { not-rain [not-A] OR not-rain [not-A] } \\
& C \rightarrow \text { not-rain [not-A] }
\end{aligned}
$$

\footnotetext{
${ }^{7}$ Here is the lynch pin of the argument. The implication operator contains a forward moving identity and a backward moving disjunction.
} 
The interpretation of this solution, according to Florensky, is that "the meaning of the truthfulness of [C] entrains the negation of $[A]$, i.e., in other words, that it is impossible to affirm $[A]$ insofar as, at the same time as, if [C] is also valid." The nature of entrainment is that attention must be paid to the context in which $A$ is spoken in order to confirm or deny truthful statements.

Suppose in our example we take $C$ to be winter. Then, the first truth claim is that rain is wet, and the second truth claim is that winter implies rain is not wet. If the starting point was the context of summer, then both claims could be taken as true if it is recognized that the second truth claim is using the term "rain" in a new context of winter in which it falls as snow and therefore is not wet. What if the initial context was all that was known in the world? Perhaps, for example, the person making the second claim comes to the equator from a northern country that experiences winter. Not having experienced winter, suppose the person living at the equator has no word or concept for snow. "Rain" then becomes the most apt word to refer to snow, although it both is and is not snow. Rain is snow in the sense that it is water falling from the sky but it is not snow in the different sense that it is not frozen like snow is. Has the person who used the word "rain" in this context spoken meaninglessly? Florensky argues no:

This judge [symbolic logic] having examined the case, pronounces a fully determinate judgement: [winter implies not-rain], i.e., in other words, without injuring either of the disputing sides with reproach of meaningless testimony and even recognizing the rightness of both, the judge affirms that neither can speak of [rain] at those times and under those conditions when [winter] is under force. The first side, affirming the condition [rain implies wet] is right; but the second side, affirming the condition [winter implies rain implies not wet] is also right. But both sides should realize that the usual, everyday, ubiquitous [rain] stops being such under special circumstances, precisely under the condition [winter]. [Florensky, 357].

It is significant to note that this logical movement involves three stases $[A, B, C]$ and not just two. Neither is it reducible to binary combinations ${ }^{8}$. Therefore, if we stay within the confines of traditional binary logic, the paradoxical claim involving $C$ is merely a contradiction that must be excluded from the initial formal system. The logic of three, however, allows for the opening up of such a closed system to what, from the perspective of the closed logical system, must be called impossible.

This opening happens through paradox. First one of the stases, in our case rain, is emptied of its determinate content that comes from the original formal system. It is then able to transgress the limit of the original formal system by forming a "pivot", as it were, that might enter into another formal system. During the pivoting movement, rain both is and is not rain. The two formal systems provide contexts, which give sense to the meaning or "content" of the stasis. In our case it was the pivot between summer and winter as "systems". In this way, two formal systems can be related to one another as juxtapositions made possible by the implicit disjunction in the logical operator $\rightarrow$ which has likewise been internalized into the pivoting stasis as paradox.

The opening is most dramatic when the original formal system is taken to be the measure of all that is true. In this case, paradox opens the system to the impossible. The opening happens by revealing an overarching context to the whole formal system that circumscribes or contains the system and its totality. The "world" is enlarged, so to speak, by the discovery that it is embedded in something beyond itself. Once the formal system is opened, what was formerly deemed "impossible" is seen to be capable

\footnotetext{
${ }^{8}$ The three stases are different in nature: the first is the same (a subject), the second is the other to the same (a quality) and the third is the systemic formal whole (context).
} 
of being brought within the realm of the possible, although this movement requires expanding the original system and its forms. What was form(er/al)ly both impossible and unspeakable enters into the realm of possibility and can be replicated or iterated to form a new stasis. This is the movement that captivated the Pythagoreans as they discovered that the real numbers are infinitely more expansive than the rational numbers. It is also the movement that Godel used to demonstrate the incompleteness theorems of number theory.

Further reading: The Priority of Relation for Creation: A primer in the logic of three.

\section{Third Harmonic Progression: Law}

Through the second harmonic progression, we found an opening within the totalizing system of RU by way of metaphor. This opening disclosed a larger context in which RU is embedded, namely the Real. The opening occurs when the contradiction of Two is resolved through a Third. The disclosure or revelation requires the irreducible logic of three and is impossible within binary logic which is the underlying process defining RU. The logic of three entrains negation and introduces disjuncture into the processes of logical reasoning. It thereby also contains RU by explicitly embedding context into the formal processes.

In binary logic there is only one context, which is formed by the totalizing system of logic. This formal context is a structure. It has the form of spatiality. And the processes of logic-the temporal--occur within the formal system giving significance to its given stases or objects. With the logic of three, there can be more than one context. For example, our case above involved the contexts of summer and winter. The context is the stasis of the formal system as a system. The context possesses interiority and that interiority comes from the stases that form the system. The context, in turn, gives form to the stases such that they have meaning or signification. In other words, the context provides the foundation for the forms and the forms contain the stases as stases. These forms are exterior images and bring about objectivity.

However, just as there is a disjuncture between two contexts, so also there can be a disjuncture within a stasis. As with binary logic, the part reflects the whole. The simplest instance of disjuncture is the binary bit which can be either/or. This disjuncture allows a given stasis to pivot between contexts as was the case with the stasis rain in our example above. The disjuncture gives the stasis interiority and is the means whereby it can pivot between formal systems ${ }^{9}$.

So, instead of dealing with the fully synchronized objects of $R U$, we are now dealing with stases which must come into synchronization to be real. The simplest instance of a stasis is the quantum bit.

A quantum bit is an interpreter.

There are three categories to the quantum. The quantum can be the locus for agency or process. This is called Firstness. The Firstness of a quantum is pure act and is particular. The quantum has form which contains it, although not fully. The form comes to the quantum from the formal system in which it is embedded as within a context. The form is general and is a property of the formal system. Therefore the quantum has form by virtue of its action within an ensemble of quanta that make up the formal system

${ }^{9}$ Through disjuncture, the stasis can be a subject. 
as context. This is called Thirdness. Finally, the quantum has Otherness. It does not rest in itself. For example, the either/or of the quantum bit is an irreducible contradiction of self-othering. Otherness comes from and allows the quantum to relate to other quanta. Otherness is relation. The Otherness of the quantum allows re-presentation such that the interiority of the quantum implies the exterior image of other quanta.

The three categories of the quantum imply that there is a difference between the quantum in its particularity (subjectivity or Firstness) and its generality (objectivity or Thirdness). The generality belongs to the formal system in which it is embedded and makes it inextricably entangled with other quanta in the ensemble forming the system. The particularity, as the locus of action, is disjointed from the generality inasmuch as the quantum can pivot into entanglement with another formal system. Such a pivot occurs through the other-ness of relationship.

Consider for example a quantum bit within the formal system of Euclidean geometry. It can enter into the system of relations with other quanta by sublating is Otherness. Such sublation is a symmetry breaking of the interiority such that the quantum has fixed orientation or handedness or "spin" and that orientation relates back the ensemble as a whole. No longer is the quantum in an indeterminate state of either/or. It is in a state of orientation or handedness that is synchronized with the whole system. Indeed it is only because all the quanta of the whole Euclidean system have sublated their otherness and entered into synchronicity, that the broken symmetry of orientation comes about which in turn stabilizes the system as a system. This broken symmetry, a habit of action among all the quanta in the ensemble, forms a geometrical law of the excluded middle creating "handedness" within the Euclidean system. Only quanta that reflect that law-whose "spin" has the mutually shared handedness - can be part of the system. From the context of the system, they constitute the real. However, the whole system is inert.

Yet the real is not inert because the broken symmetry of orientation is a general form belonging to the ensemble and the quantum maintains its ability to pivot by virtue of its interiority. It is as if the quantum's interiority has been collapsed by the formal system into a fixed state. The quantum has interpreted the formal system. But this collapse or interpretation is neither final nor eternal because the quantum remains a locus of potential action. The potential action is the ability to pivot either/or between the given orientation and its opposing Other. Such pivoting would involve the quantum coming into a relation with another formal system, different from the first in its particularity although the same as the first in its generality. Through the disjointed relationship between these two formal systems the quantum can communicate orientation, its interpretation, as an external form.

The mediation of this process is light which obeys the logic of three.

We arrive at the consideration of light as a transcendental movement of entanglement which allows the creation of disjointed formal systems. The formal systems constrain quanta as processes by giving them structural form. Quanta have exteriority in the form of images or states. They also have interiority as indeterminate juxtaposition. The quanta can take on fixed images by following the laws or rules or habits of the ensemble forming the system or context. However, they also remain capable of potential action that changes their interior state and in so doing brings the interior into exterior representation. Through these processes quanta interpret the real.

Further reading: A Practical Guide to Relational Ontology in Modern Physics with an Annotated Bibliography. 


\section{Decrescendo}

Law is not the highest category. It is purely formal and general. The real, by contrast, is particular. Law creates external form that applies to an ensemble of entangled quanta. It is a property of the ensemble that gives form to each constituent quantum. The ensemble of quanta work out these forms as relational processes of interpretation. The law of the excluded middle applies to formal systems and this law is what gives stability and structure. Formal systems create context but context can always be transcended and brought into relation with other contexts. This transcendental movement is called Light.

Further reading: Word, Identity and the Relational Form of the Individual in Paul's Letter to the Romans.

\section{Coda: The Entrainment of Negation}

The movement beyond RU has led us to the logic of inference by way of the entrainment of negation. Whereas the binary logic of RU involves a closed system of lawful determination, the logic of inference involves the open and creative fulfilment of law. Inference is an interpretive process ${ }^{10}$.

The logic of inference is the logic of signs. When we say $\mathbf{A}$ implies $\mathbf{B}$ (that is to say: if $\mathbf{A}$ is true, then $\mathbf{B}$ is true), we are also asserting that $\mathbf{A}$ can be taken as a sign of $\mathbf{B}$.

There are two ways we might represent inference within traditional formal logic.

$\begin{array}{ll}\text { Not-A OR B } & \text { (subjective representation) } \\ \text { Not-(A AND Not-B) } & \text { (objective representation) }\end{array}$

These two ways are complementary in the following sense. The first representation is based on the relational operator $O R$ and manifests the subject of succession which is metaphorically temporal. The second representation is based on the relational operator AND and manifests the object of co-presence which is metaphorically spatially ${ }^{11}$.

Within these representations of inference, relation is prior to stasis. Inference takes us from A to B while allowing that both $\mathbf{A}$ and $\mathbf{B}$ are, in themselves, indeterminate. This has subtle implications for how we might understand the logic of inference. As discussed by Kauffman, the inferential operation that relates $\mathbf{A}$ and $\mathbf{B}$ is not a disjointed conjunction of two discrete elements or stases as would be the case in

\footnotetext{
${ }^{10}$ While traditional binary logic is the explicated logic of Two, inference is the explicated logic of Three. This moves us from thinking about numbers in terms of assemblages of discrete finite units within spatialized structures, to thinking about numbers in terms of formal principles of spatio-temporal processes. In The Mathematics of Charles Sanders Peirce, Kauffman explores the logic of inference and draws out several formal principles that are taken up in this section of the paper.

${ }^{11}$ For further exploration of the "metaphysics" of AND and OR, see Otherwise than Being, or Beyond Essence by Emmanuel Levinas.
} 
traditional binary logic. The inferential operator is, what Kauffman calls, a Portmanteau sign in which a single sign holds two meanings. "A Portmanteau word is a holder of two or more words, each justly truncated to fit the truncate of the other." Specifically the Portmanteau sign of inference is the Sign of Illation as described by Peirce. It can be represented by fusing a horizontal overbar representing negation of the sign beneath it with a plus symbol representing the conjunction of two signs through OR.

Sign of Illation or inference

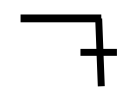

The Sign of Illation relates two signs by way of logical inference:

Not-A OR B

\section{A}

This sign signifies the relation of an antecedent to a consequent: from $\mathbf{A}$ follows $\mathbf{B}$. It is a directed relation in the sense that the positions of the two related signs cannot be interchanged as would be the case with equality. The Sign of Illation represents succession or progression as a mediated and directed relation between two stases without beginning or end and in this sense is similar to Derrida's notion of différance.

The Sign of Illation entrains the operator Not such that it is subsumed within systems of relations. Following Peirce's interpretation, "To say that A is false, is the same as to say that from an antecedent follows any consequent that we like. This is naturally shown by leaving a blank space for the consequent, which may be filled in at pleasure" [Peirce as quoted by Kauffman]. Thus, we can represent "A is false" as below.
A is false
A t

This representation of falseness presents $\mathbf{A}$ as a sign of nothing. One way to think about this is to recognize that "nothing" is a word that refers to that which does not exist. But if something does not exist, it cannot be referred to-there is an explicit contraction involved in defining "nothing". In fact, when we speak about nothing, we are likely referring to something which is absent, something which has the potential to exist but does not actually exist. The recognition of absence comes from the relational system in which absence is contextualized. Absolute nothingness, without relation to something else, cannot be represented ${ }^{12}$.

\footnotetext{
12 It may seem confusing for me to signify "Absolute nothingness" after stating that it does not exist and that the signification is a logical contradiction. What is happening, I think, is that we are coming into contact with a
} 
The representation of "A is false" also presents $\mathbf{A}$ as a final cause or end in itself with no further consequent. Within the logic of inference, a final cause cannot be represented. All representable entities exist in some manner of causal relation to other entities and all are signs that signify something beyond themselves ${ }^{13}$.

The Sign of Illation allows for a pivot duality which can translate signs between different contexts. To see how a pivot works, suppose we have a closed system of inference such as Number theory. Let's call the system of inference $\mathbf{C}$, where $\mathbf{C}$ establishes the context for interpreting the signs. Within the system $\mathbf{C}$ we might represent a false statement $\mathbf{A}$ as below.

A is false for $\mathbf{C}$

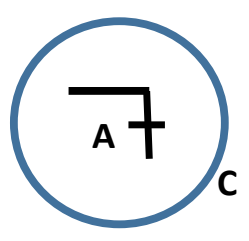

The system $\mathbf{C}$ constrains the negation of $\mathbf{A}$ such that it refers to an absence within a system of relations and it does not refer to an absolute state (which cannot be represented).

At the same time, the negation of $\mathbf{A}$ frees up the sign such that it now has the potential to refer outside of the context $\mathbf{C}$. But in order to actualize this potential, it must become embedded in a second context $\mathbf{C}^{\prime}$ which is other than $\mathbf{C}$.
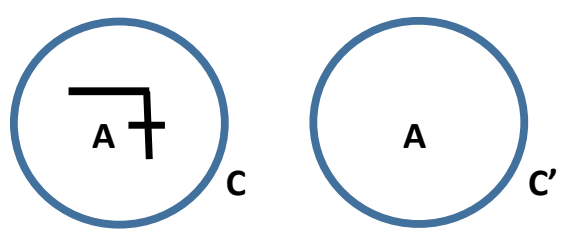

This pivoting movement involves indetermination, freedom, choice. It is a creative impulse. Yet the creative impulse does not overcome falseness until it is received or completed or fulfilled by bringing the two contexts into an overarching context C" in which both contexts are synchronously true.

transcending movement beyond the realm of representable existence. "Absolute nothingness" might then better be taken as referring to an internal state of receptivity to something beyond the world of representations, a creative potential on the threshold of actualization, a movement from darkness into light. A primal beginning. ${ }^{13}$ No existent can be a sign of "nothing". Every thing is a sign of something else. The web of significations, the representable world, has is fulfillment in a unifying final cause that draws all things into itself. In the beginning was the Word. 


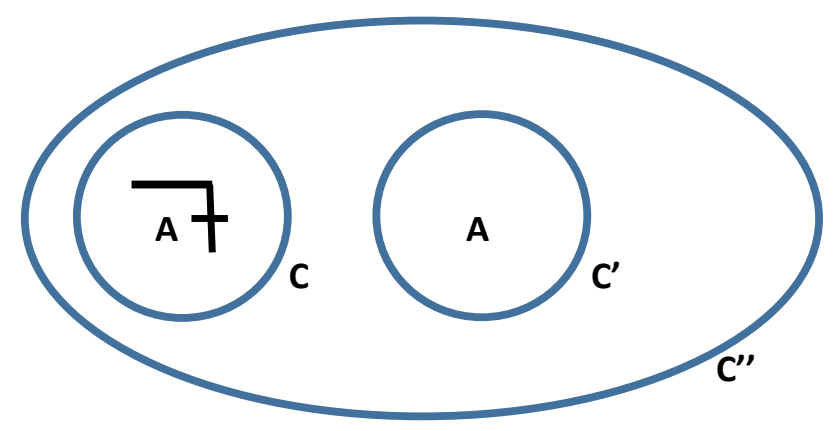

Within this overarching context $\mathbf{C}^{\prime \prime}$, we would have the representation:

\section{A}

That is to say, $\boldsymbol{A}$ implies $\boldsymbol{A}$ which is true for any $\mathbf{A}$.

But now $\mathbf{A}$ can be a pivot sign that can take on two mutually opposing values whose meaning is determined by the way in which the two contexts $\mathbf{C}$ and $\mathbf{C}^{\prime}$ are united in the third $\mathbf{C}^{\prime \prime}$.

Suppose $\mathbf{C}^{\prime \prime}$ is the context in which $\mathbf{A}$ cannot be false in both $\mathbf{C}$ and $\mathbf{C}^{\prime}$. Then $\mathbf{C}^{\prime \prime}$ can be represented as below.

\section{Not-( Not-A in C and Not-A in C')}

This is the inference that

\section{If Not-A in C, then A in C'}

The contexts can be synchronized if $\mathbf{A}$ is a sign that can take on one of two opposing values and whatever value $\mathbf{A}$ takes on in context $\mathbf{C}$ it takes on the opposite value in context $\mathbf{C}^{\prime}$.

But now $\mathbf{A}$ has assumed a new dimension of depth. In traditional binary logic, the sign $\mathbf{A}$ would be taken to refer univocally to a single state-A would be fully explicated as an actual object. Within our more expansion logic of inference, $\mathbf{A}$ has interiority such that it could be in one of two possible states depending on the way in which two contexts are synchronized. A can be either subject or object according to potential agency and actual realization, respectively.

Following Peirce, we can represent this graphically by taking a circle or simple closed curve drawn around a sign. The circle makes a distinction between its inside and its outside and thereby represents distinction [Kauffman]. 
A circle drawn around a sign changes the enclosed assertion into a negative. So, for example, a circle drawn around $\mathbf{A}$ asserts the negation of $\mathbf{A}$.

Not-A

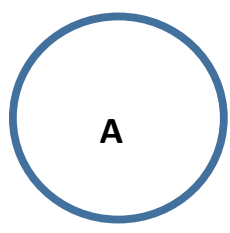

We can apply Peirce's "existential graphs" to our discussion by taking the circle to also represent context. Then the above graph tells us: When A exists in isolation within a context where the context also exists in isolation, then not- $A$. This is equivalent to the assertion " $A$ is false" that we arrived at using the Sign of Illation involving an overbar fused with a cross.

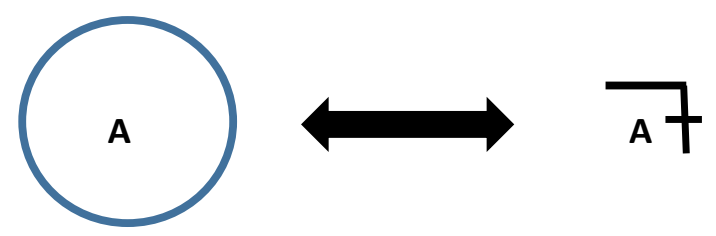

The existential graphs can also represent the Sign of Illation. Recall $A$ implies (is a sign of) $B$ is logically equivalent to Not-(A AND Not-B) in the objective representation.

Not-(A AND Not-B)

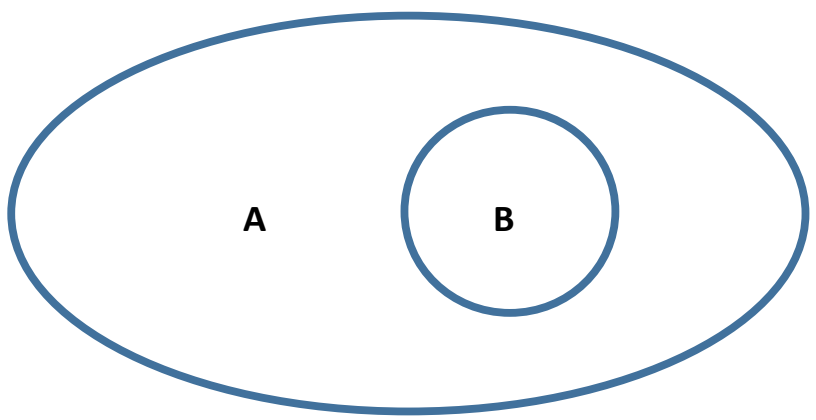

Then, using the existential graphs, the two contexts $\mathbf{C}$ and $\mathbf{C}^{\prime}$ contexts become synchronized when the circle representing any context is taken to mean that the enclosed assertion is negated. 


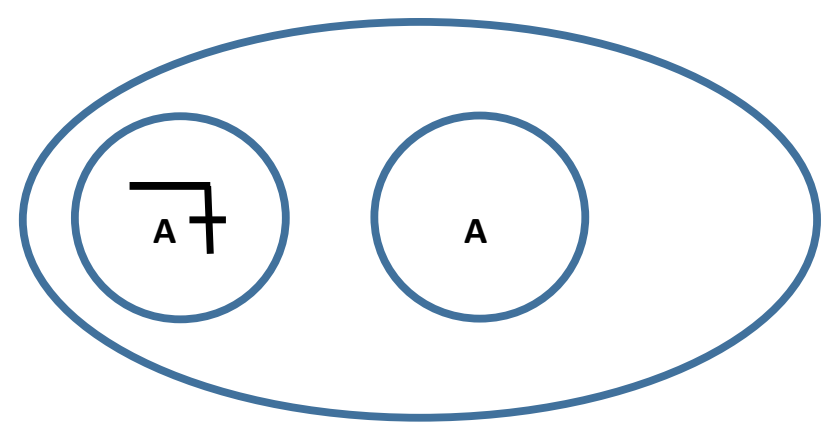

But for the simple case of opposition ${ }^{14}$, we have Not-Not-A is $\mathbf{A}$.

Therefore we can substitute $\mathbf{A}$ for the graph below.

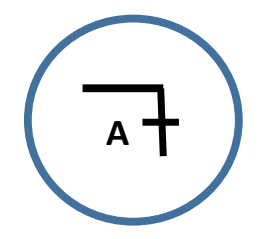

This leaves us with the following simplified graph representing the synchronized contexts

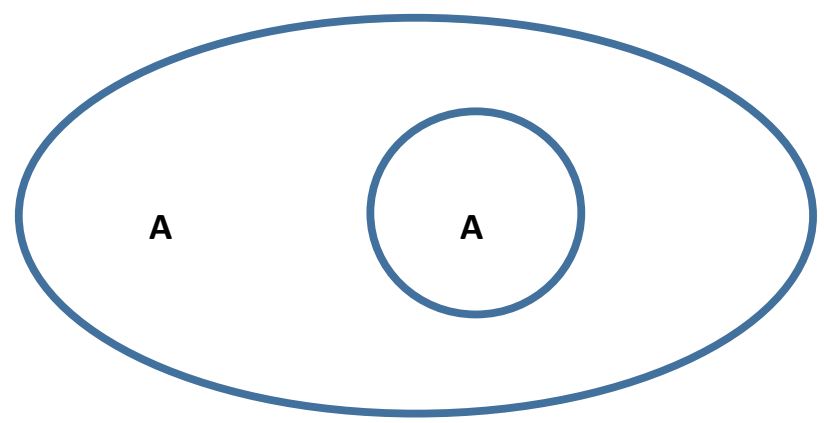

This is the logic of identity. $\mathbf{A}$ implies $\mathbf{A}$, where $\mathbf{A}$ is a pivot symbol that can take on one of two opposing values in a given context such that it takes on the opposite value in a second context that has been synchronized with the first context. Within this logic of identity, $\mathbf{A}$ is the same according to external form that comes from the context, yet it is distinguished according to internal value. It is a fully relational entity that only has form and manifestation within a context and which has agency to pivot by virtue of its relation to an opposing or complementary image of itself that is relationally united to itself by way of interiority.

\footnotetext{
${ }^{14}$ The case of opposition (duality) is also implicit in the use of two-dimensional planar circles as the formal basis for existential graphs.
} 
This is also the representation of two coupled quantum bits. And quantum mechanics might therefore be interpreted as the embodiment of the logic of inference.

Further reading: On the Foundations of Physics: An entrance into formal semiotics.

\section{References}

Florensky, Pavel. The Pillar and Ground of the Truth: An essay in orthodox theodicy in twelve letters. Transl by Boris Jakim. New Jersey: Princeton University Press, 1997.

Gallagher, Timothy M. The Discernment of Spirits: An Ignatian guide for everyday living. New York: Crossroad Publishing Company, 2005.

Habermas, Jurgen. The Theory of Communicative Action, 1981.

https://en.wikipedia.org/wiki/The Theory of Communicative Action

Kauffman, Louis H. The Mathematics of Charles Sanders Peirce. In Cybernetics \& Human Knowing. 8(1-2), 79-110, 2001.

Kerruish, Valerie and Petersen, Uwe. Philosophical Sanity, Mysteries of the Understanding, and Dialectical Logic. [Available Aug 8 2018:

https://www.academia.edu/20791477/Philosophical Sanity Mysteries of the Understanding and Dia lectical Logic ]

Kruse, Felicia. Is cosmic evolution semiosis? In From Time and Chance to Consciousness: Studies in the metaphysics of Charles Peirce, Eds. Moore, Edward and Robin, Richard, 1994. [Available Aug 82018 : https://www.academia.edu/7104060/Is Cosmic Evolution Semiosis ]

Levinas, Emmanuel. Otherwise than Being, or Beyond Essence. Translated by Alphonso Lingis. Pittsburgh: Duquesne University Press, 1974. https://en.wikipedia.org/wiki/Otherwise than Being

Marion, Jean-Luc. Givenness and Revelation. Transl by Stephen E Lewis. Oxford: Oxford University Press, 2016.

Unger, Roberto Mangabeira and Smolin, Lee. The Singular Universe and the Reality of Time: A proposal in natural philosophy, 2015. https://en.wikipedia.org/wiki/The Singular Universe and the Reality of Time

Whitehead, Alfred North and Russel, Bertrand. Principia Mathematica, 1910-13. https://en.wikipedia.org/wiki/Principia Mathematica

Wittgenstein, Ludwig. Tractatus Logico-Philosophicus, 1921 https://en.wikipedia.org/wiki/Tractatus Logico-Philosophicus 